\title{
Formation of quark phases in protoneutron stars: the transition from the 2SC to the normal quark phase
}

\author{
G. Pagliara \\ Institut für Theoretische Physik, Ruprecht-Karls-Universität, \\ Philosophenweg 16, D-69120, Heidelberg, Germany
}

\begin{abstract}
We study the process of formation of quark phases in protoneutron stars. After calculating the phase transition between nucleonic matter and the 2SC phase at fixed entropy and lepton fraction, we show that an unpairing transition between the 2SC phase and the normal quark phase occurs for low lepton fractions. We then calculate the process of diffusion of neutrinos in protoneutron stars and show that for intermediate values of the mass of the star, the deleptonization triggers the phase transition between the two quark phases after a temporal delay of a few seconds. In less massive stars instead only the normal quark phase is formed at the end of the deleptonization stage. We also discuss the possible astrophysical implications of our scenario.
\end{abstract}

\section{INTRODUCTION}

In the last years, a new very exciting theoretical discovery in QCD concerns the low temperature and high density region of the phase diagram of strongly interacting matter where quarks form a state of color superconductivity. In particular, the so called Color-Flavor-Locking (CFL) phase in which all quarks are paired, was shown to be the ground state of strongly interacting matter at asymptotically high densities [1]. In turn, this opens the possibility that this new quark phase appears in the core of neutron stars and affects their properties. A number of studies has been done to investigate the structure of the QCD phase diagram for the conditions that are realized in neutron stars: the requirements of charge neutrality and equilibrium with respect to weak interactions, which must be fulfilled in compact star matter, and the finite value of the mass of the strange quark split the values of the chemical potentials of the different quark flavors thus allowing for the existence of many color superconducting phases in the phase diagram as the 2SC phase, the normal (unpaired) phase, the gapless phases and the crystalline phase [2 6]. An important question concerns when and how these quark phases are eventually formed in a neutron star. In [2, 7, 9] it was argued that while the 2SC phase or the normal quark phase might be formed already during the protoneutron star stage i.e. when matter is hot and lepton rich, the CFL phase could appear only later when the star has almost completed its deleptonization through the emission of neutrinos. The possibility of forming different quark phases during the protoneutron stars evolution could have very intriguing observable consequences as proposed in [10 13] in connection with gamma-ray-bursts.

To date, there are no detailed numerical simulations of the process of formation of color superconducting phases in protoneutron stars: such a calculation is indeed very complicated because, besides the equation of state, it involves also the computation of the neutrino mean free path in the different quark phases and it requires to solve numerically the transport equations describing the diffusion of neutrinos within the protoneutron star matter.
A first preliminary study was presented in [14], where, besides the calculation of the neutrino mean free path in color superconducting quark matter, a simple modeling of the neutrino cooling was presented in order to discuss the possible observable signatures of the formation of a color superconducting phase in the star. The scenario consists of a second order phase transition from the unpaired phase to a superconducting phase: during the cooling, the temperature decreases and at some point reaches the critical temperature and the peak of the specific heat associated with the phase transition is met. The authors argue that the phase transition would lead to a slower cooling of the star for temperatures close to the critical temperature with possible effects on the neutrino luminosity. This possibility could be indeed realized in protoneutron stars if the color superconducting gap is of the order of a few tens of $\mathrm{MeV}$ which lead to critical temperatures comparable with the temperatures of protoneutron stars.

Here we will investigate a different scenario: at birth a protoneutron star has a lepton fraction of the order of $Y_{L}=0.4$ with a corresponding electron/proton fraction of $Y_{e}=Y_{p} \sim 0.3$ at the center of the star. The subsequent deleptonization causes a decrease of lepton and also electron fractions: consequently also $Y_{p}$ decreases and matter becomes more and more isospin asymmetric. If quark matter is present in the core of the star at birth, it is likely to be in the 2SC phase because matter is not very isospin asymmetric i.e. the number of up and down quarks are quite similar and the 2SC pairing is favored. As the deleptonization proceeds, the gradual increase of the isospin asymmetry causes a stress on the Cooper pairs and a first order phase transition from the 2SC to the unpaired quark phase is triggered at some critical value of the asymmetry [7, 15 18]. Remarkably, the phase transition between the superconducting state and the normal state in asymmetric fermionic systems has attracted much attention in the last years also in connection with cold atoms experiments (see for instance [19, 20]). By solving the neutrino diffusion equation coupled with a quasi-static temporal evolution of the structure of the star, we will show here that such a phase transition be- 


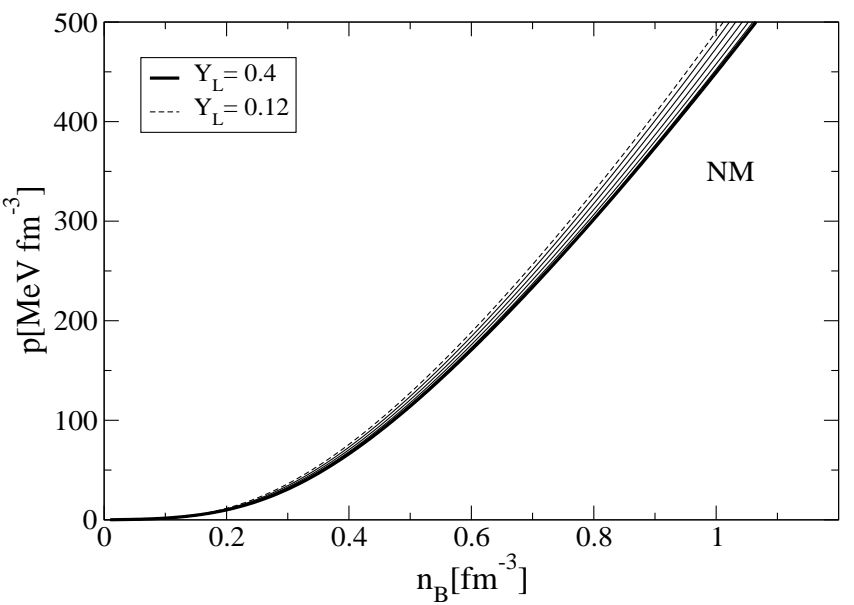

FIG. 1: Pressure as a function of the baryon density for nucleonic matter for values of $Y_{L}$ ranging from 0.4 (thick line) to 0.12 (dashed line) with intervals of 0.04 (solid lines). At lower lepton fractions, which implies also lower electron and proton fractions, the nucleonic equation of state is stiffer because of the increasing contribution of the symmetry energy to the EoS.

tween quark phases could indeed occur in protoneutron star matter and possibly gives some observational effects in the neutrino signal.

The paper is organized as follows: in Sec. II we calculate the Equation of State (EoS) for protoneutron star matter by including the pure quark and nucleonic phases and the mixed phase. In Sec. III we present and discuss the results of our numerical simulation of the neutrino diffusion and finally in Sec. IV we draw our conclusions.

\section{EQUATION OF STATE OF PROTONEUTRON STAR MATTER}

Matter in protoneutron stars is hot and lepton rich. At birth, the initial conditions correspond, to good approximation, to uniform values of the entropy per baryon $S / N=1$ and the lepton fraction $Y_{L}=0.4$ [21, 22]. Since the deleptonization reduces the value of $Y_{L}$, we will calculate the EoS for $Y_{L}$ varying within the range $0.04-0.4$. As we will explain in the next section, we disregard here the effect of reheating of the star during deleptonization and we will therefore keep $S / N$ fixed during the simulation. To describe the phase transition from Nucleonic Matter (NM) to Normal Quark matter (NQ) or 2SC matter, we use, as customary, two models: for the low density nucleonic phase we adopt the relativistic mean field model with the parameterization TM1 [23] and for the high density quark phase a modified bag model which includes also color superconductivity in the 2SC phase used in [15]. We limit our discussion here to two flavor quark matter, with massless up and down quarks and we fix the model parameters as follows: the intermediate value of the diquark coupling is considered $G_{D}=3 / 4 G_{S}$ where

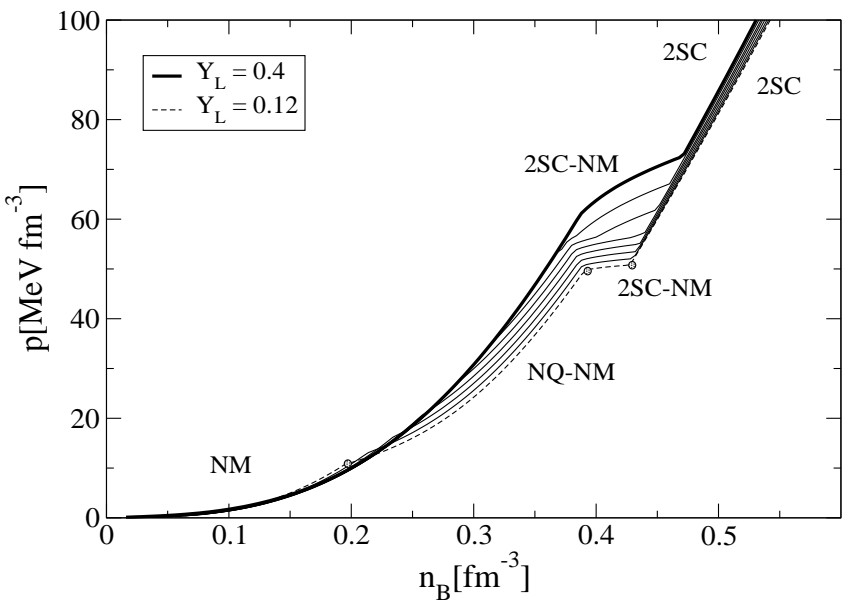

FIG. 2: Pressure as a function of the baryon density for the nucleonic matter - quark matter phase transition for values of $Y_{L}$ ranging from 0.4 (thick line) to 0.12 (dashed line) with intervals of 0.04 (solid lines). The dots on the dashed line indicate the onset and the end of the mixed phases of nucleonic matter and normal quark matter and nucleonic matter and 2SC phase. At large values of $Y_{L}$, the quark phase corresponds to the 2SC phase. At lower $Y_{L}$, the mismatch between the chemical potentials of up and down quarks is such that the pairing is broken and the normal quark phase is obtained.

$G_{S}$ is the scalar coupling and the bag constant $B$ is fixed to $155 \mathrm{MeV}^{1 / 4}$. We also include, in an effective manner, the corrections to the quark pressure due to perturbative strong interactions [24, 25]: the constant $c$, which simulates perturbative interactions, is fixed to a value of 0.05 for which we obtain a maximum mass for cold and catalyzed hybrid stars of $1.94 M_{\odot}$, in agreement with the very recent measurement of a compact star having a mass of $1.97 \pm 0.04 M_{\odot}$ [26]. For this choice of parameters, the onset of the phase transition occurs at twice nuclear saturation density for the initial lepton fraction and entropy configurations. The phase transition is computed by using the Gibbs construction as described in 21, 22, 27-29] with the conditions of global conservation of baryonic number, lepton number and electric charge. The resulting extended mixed phase is obtained by solving the following equations:

$$
\begin{array}{r}
p^{H}\left(\mu_{B}^{H}, \mu_{C}^{H}, \mu_{L}^{H}, T^{H}\right)=p^{Q}\left(\mu_{B}^{Q}, \mu_{C}^{Q}, \mu_{L}^{Q}, T^{Q}\right) \\
(1-\chi) n_{C}^{H}+\chi n_{C}^{Q}-n_{e}=0 \\
n_{e}+n_{\nu}=Y_{L} n_{B} \\
(1-\chi) s^{H}+\chi s^{Q}=S / N n_{B} \\
T^{H}=T^{Q} \\
\mu_{B}^{H}=\mu_{B}^{Q}, \mu_{C}^{H}=\mu_{C}^{Q}, \mu_{L}^{H}=\mu_{L}^{Q}
\end{array}
$$

where $p^{i}, \mu_{B}^{i}, \mu_{C}^{i}, \mu_{L}^{i}, T^{i}, n_{C}^{i}, s^{i}$ are the pressure, baryon chemical potential, charge chemical potential, lepton chemical potential, temperature, electric charge density and entropy density of the hadronic $(i=H)$ and the quark phases $(i=Q) ; n_{e}$ and $n_{\nu}$ are the electron and electron neutrino densities, $n_{B}$ is the baryon density and 
$\chi$ is the volume fraction of the quark phase. It has been recently pointed out that another possible hadron-quark mixed phase could appear in protoneutron stars if local charge neutrality and global lepton number conservation are imposed [30]; we will not consider this possibility here.

In Fig. 1, we show the EoS (pressure vs baryon density) of nucleonic matter for different values of $Y_{L}$. Notice that by decreasing $Y_{L}$, the EoS becomes stiffer: despite the fact that at lower $Y_{L}$ the contribution to the total pressure of leptons is smaller, the lower values of $Y_{e}$ implies a larger isospin asymmetry of nucleonic matter. The effect of the symmetry energy thus dominates over the reduced leptonic pressure and the EoS is stiffer. This a well known fact and it also implies that the maximum baryonic mass of protoneutron stars is smaller than the maximum baryonic mass of cold neutron star (if nucleonic matter is considered) [31].

Let us discuss now our results for the mixed phase. We show the EoS in Fig. 2: at a large lepton fraction, $Y_{L}=0.4$, after a low density nucleonic phase, the 2SCNM mixed phase starts and at high density the pure 2SC phase takes place. By decreasing the value of $Y_{L}$, at some point, close to the onset of the mixed phase, the 2SC pairing cannot take place anymore because of the large mismatch between the up and down chemical potentials (the isospin density increases as $Y_{L}$ decreases) and the normal quark phase starts to form. Notice that the transition from the 2SC phase to the normal phase occurs close to the onset of the mixed phase and moves to higher densities as $Y_{L}$ is further decreased. This is due to the fact that the local isospin density of the quark phase is large at the onset of the mixed phase and gradually decreases as the volume fraction increases, as shown in Refs. 15, 32, 33]. The largest stress on the up and down chemical potentials is thus realized close to the onset of the mixed phase: the hadron quark phase transition induces also a phase transition between quark phases. To calculate the transition between the NQ and the 2SC phases within the mixed phase we use the following simple prescription: we start with the high density pure $2 \mathrm{SC}$ phase, $\chi=1$, and we decrease the density until we reach the border of the mixed phase with the NM obtained by solving the system of Eqs. 1-6; we solve the same system at decreasing values of the baryon density and $\chi$ and obtain the solutions $\mu_{B}^{*}, \mu_{C}^{*}, \mu_{L}^{*}$ and $T^{*}$; then we accept the solution of the system only if the pressure of the normal quark phase computed at the same $\mu_{B}^{*}, \mu_{C}^{*}, \mu_{L}^{*}$ and $T^{*}$ is lower than the pressure of the $2 \mathrm{SC}$ phase. When this condition is not fulfilled anymore we choose the NQ phase as the quark component of the mixed phase. This is a simple way to describe the unpairing phase transition between the 2SC and the NQ phases (similar to a Maxwell construction) within the mixed phase with NM. Actually, since this phase transition is also of first order one should introduce two volume fractions associated with the two quark phases $\chi_{N Q}$ and $\chi_{2 S C}$ and solve a more complicated system of equations which provides a

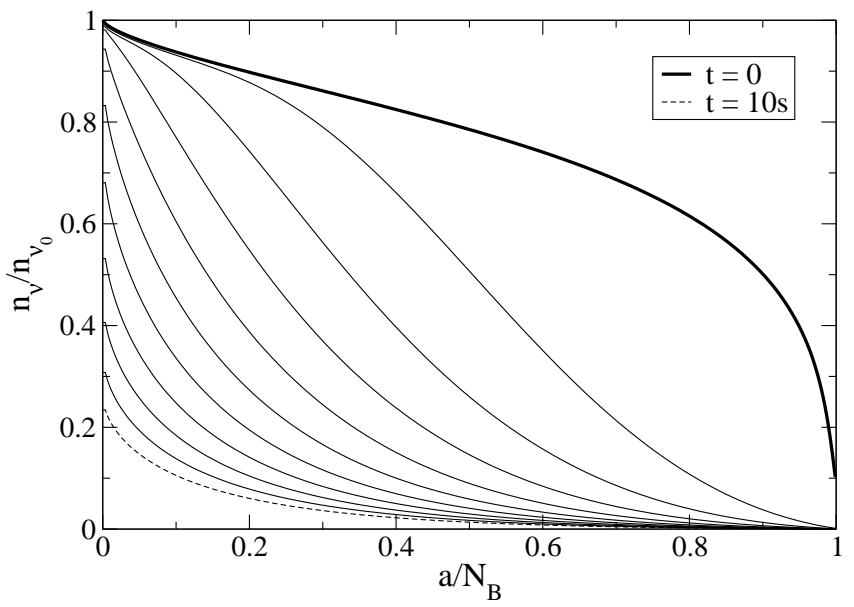

FIG. 3: Temporal variation of the neutrino density as a function of the enclosed baryonic number for a neutron star of total baryonic number $N_{B}=2.29 \times 10^{57}$. The initial central neutrino density at the center of the star, $n_{\nu_{0}}=1.88 \times 10^{-2} \mathrm{fm}^{-3}$. The thick line corresponds to the initial configuration, $t=0$, and the dashed line to the configuration at $t=10 \mathrm{~s}$. The thin solid lines stand for the intermediate configurations with temporal steps of $1 \mathrm{~s}$.

mixed phase made of three components. A similar calculation has been done, for cold and beta stable matter, for mixed phases composed by quark color superconducting phases [34], but calculations for protoneutron star matter, also including the nucleonic phase, have never been performed. Our procedure, while simple, is sensible since the NQ-2SC phase transition is not a strong first order, i.e. the jump of the density is not large when going from one phase to the other one (the density of the 2SC phase is larger than the one of the NQ by an additive term scaling as $(\Delta / \mu)^{2}$, with $\Delta \sim 100 \mathrm{MeV}$ being the superconducting gap). Therefore the three components mixed phase would actually cover a small interval of density. We consider a full calculation with the three components as an interesting future development of this work.

\section{NEUTRINO DIFFUSION IN PROTONEUTRON STARS}

The evolution of a protoneutron star can be schematically divided into two separate stages [31]: the deleptonization, during which the trapped neutrinos diffuse and are gradually released from the star, and the cooling, during which the entropy of the star reduces by the emission of free streaming neutrinos. During deleptonization, the star is actually reheated by the interactions of neutrino with baryonic matter and the initial $S / N \sim 1$ raises to a value of $\sim 2$. This is for us the most interesting stage because $Y_{e}$ and $Y_{p}$ also decrease during it thus leading to a sizable change of the chemical composition of the star which, as we will show, is responsible for the formation of different quark phases. The stan- 


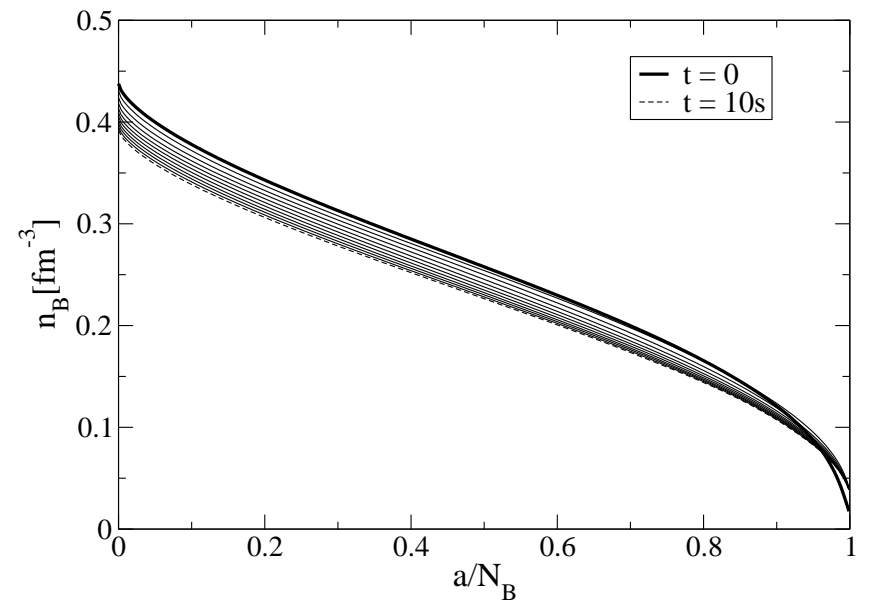

FIG. 4: Temporal variation of the baryon density as a function of the enclosed baryonic number for a neutron star of total baryonic number $N_{B}=2.29 \times 10^{57}$. The thick line corresponds to the initial configuration $t=0$ and the dashed line to the configuration at $t=10 \mathrm{~s}$. The thin solid lines stand for the intermediate configurations with temporal steps of $1 \mathrm{~s}$. A small decrease of the central density occurs during deleptonization.

dard technique to simulate the evolution of protoneutron stars consists of solving two partial differential equations associated with the transport of lepton number and the transport of energy within the so called diffusion approximation 35 38. These equations are coupled with the Tolman-Oppenheimer-Volkoff (TOV) equations to take into account the mechanical readjustment of the star during the evolution. Indeed, the time scales of diffusion are much larger than the dynamical time scale of the compact objects and therefore the evolution of the structure of the star follows quasi-static equilibrium configurations. In the cases in which the deleptonization drives a collapse of the star, because of the formation of exotic phases for instance, the hydrodynamical evolution must be also taken into account as done in [39] or more recently in [40, 41] where for the first time consistent simulations from the core collapse of the supernova to the cooling epoch of the newly formed neutron star have been performed.

The crucial ingredients for such calculations are the EoS and the cross sections of all the possible interactions of neutrinos with the matter of the star. Different studies have addressed the possibility to form "exotic" phases during the evolution of a protoneutron star as hyperons [37, 38], kaon condensates [42] or finally normal quark matter [43]. A similar calculation taking into account color superconducting phases is still missing and this is the problem that we want to address in this paper. Since we want here to provide a qualitative description on how the unpairing transition could occur in a compact star, we will adopt the following simplifying assumptions in the treatment of the neutrino transport in protoneutron stars: we will consider only the first seconds of the stage of deleptonization when neutrinos are still degener-

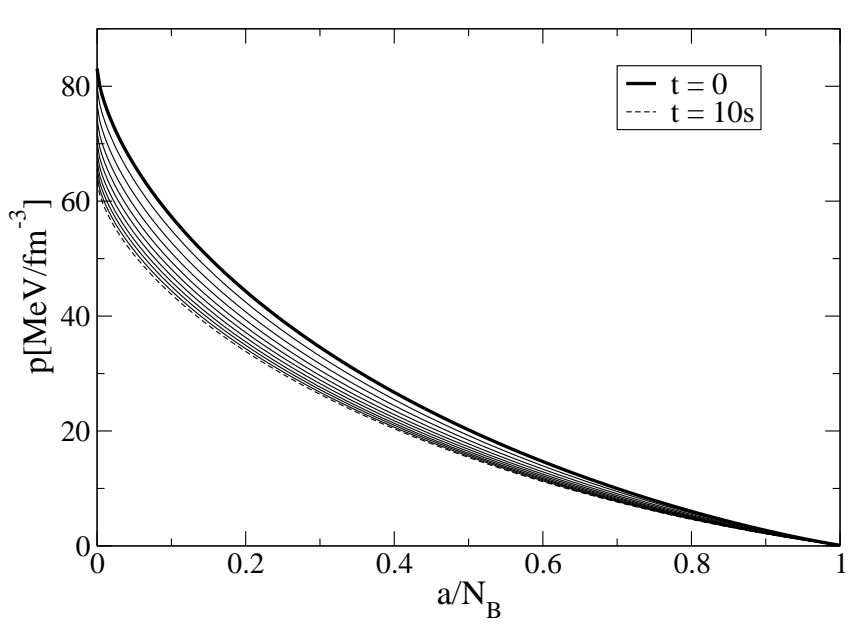

FIG. 5: Temporal variation of the pressure as a function of the enclosed baryonic number for a neutron star of total baryonic number $N_{B}=2.29 \times 10^{57}$. The thick line corresponds to the initial configuration $t=0$ and the dashed line to the configuration at $t=10 \mathrm{~s}$. The thin solid lines stand for the intermediate configurations with temporal steps of 1s. A small decrease of the central pressure occurs during deleptonization.

ate and we will assume the entropy profile to be constant during the evolution $(S / N=1)$. These assumptions lead to several simplifications: we will not solve the equation for the energy transport but just the one describing the lepton number transport coupled with the TOV equations; when neutrinos are degenerate the dominant contribution to the mean free path is given by the absorption processes of neutrinos by non-degenerate neutrons in NM and by degenerate quarks in the quark phases [31, 44]. Moreover, also regarding the EoS to be used as input for the simulations we will use a two-dimensional table with the baryon density and the lepton fraction as independent variables and the ratio $S / N$ will be kept constant. Because of our simplifying assumptions, our calculation has two important limitations: the estimate for the timing of the phase transition must be taken only as an order of magnitude estimate; indeed, because of the reheating that we are neglecting here, all the cross sections of neutrinos would be enhanced by the increase of the temperature and therefore we expect the global evolution of the system to be slower than what we find here. Secondly, we cannot provide here an estimate of the neutrino luminosity which is obtained by solving the energy transport equation.

Let us discuss now the equations that we solve to simulate the deleptonization [37]:

$$
\begin{aligned}
n_{B} \frac{\partial Y_{L}}{\partial t} & =\frac{\Gamma}{r^{2}} \frac{\partial}{\partial r}\left(r^{2} e^{\phi} \frac{\lambda}{3} \frac{\partial n_{\nu_{e}}}{\partial r}\right) \\
\frac{d p}{d r} & =-(p+e) \frac{m+4 \pi r^{3} p}{r^{2}-2 m r} \\
\frac{d m}{d r} & =4 \pi r^{2} e
\end{aligned}
$$




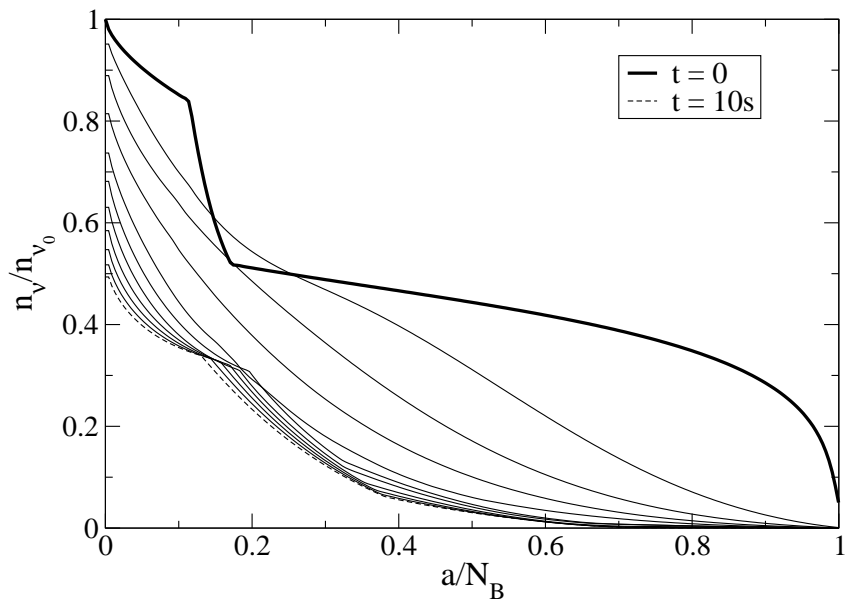

FIG. 6: Temporal variation of the neutrino density as a function of the enclosed baryonic number for a hybrid star of baryonic number $N_{B}=2.29 \times 10^{57}$. The initial central neutrino density, $n_{\nu_{0}}=3.44 \times 10^{-2} \mathrm{fm}^{-3}$. The thick line corresponds to the initial configuration $t=0$ and the dashed line to the configuration at $t=10 \mathrm{~s}$. The thin solid lines stand for the intermediate configurations with temporal steps of 1s.

$$
\begin{aligned}
& \frac{d a}{d r}=\frac{4 \pi r^{2} n_{B}}{\sqrt{1-2 m / r}} \\
& \frac{d \phi}{d r}=\frac{m+4 \pi r^{3} p}{r^{2}-2 m r}
\end{aligned}
$$

where $r$ is the radial distance in spherical coordinates, $\Gamma=\sqrt{1-2 m / r}$, and $e^{\phi}$ are the general relativity corrections to the diffusion equation, $\lambda$ is the spectral average of the electron neutrino mean free path, $p, e, m$ and $a$ are the pressure, the energy density, the enclosed gravitational mass and the enclosed baryonic number (we use the gravitational units $G=1$ and $c=1$ ). The first equation describes the diffusion of neutrinos driven by the neutrino density gradient (i.e. within the diffusion approximation ) and the last four equations describe the mechanical equilibrium of the star, the TOV equations. The initial condition and the boundary conditions are specified as follows: for the initial condition we use an EoS with constant $S / N=1$ and $Y_{L}=0.4$; on the boundaries, $r=0$ and $r=R$ (where $R$ is the radius of the star which, numerically, corresponds to a very small value of the pressure), the neutrino flux $F(r)=-\frac{\lambda}{3} \frac{\partial n_{\nu_{e}}}{\partial r}$ is taken as $F(0)=0$ and $F(R)=f n_{\nu_{e}}$ where $f$ is a constant varied between $0.1-0.6$. The boundary condition at $r=R$ basically imposes that the flux of neutrinos is a certain fraction $f$ of the flux that one would have if neutrinos were free streaming [38]. We also need initial conditions for the TOV equations: the central pressure is chosen arbitrarily, $p(r=0)=p_{c}$ (different values of $p_{c}$ lead to different values for the total baryon number of the star), $m(r=0)=0, a(r=0)=0$ and $\phi(r=R)=\frac{1}{2} \log (1-2 m / r)$. Finally, concerning the mean free paths, during the initial stage of the evolution, the absorption processes are the dominant ones and thus we take for NM the mean free path associated with the absorption of degenerate neutrinos by non-degenerate neutrons [31]:

$$
\lambda_{n}=\frac{4}{n_{n} \sigma_{0}\left(1+3 g_{A}^{2}\right)}\left(\frac{m_{e}}{E_{\nu}}\right)^{2}
$$

where $n_{n}$ is the neutron number density, $\sigma_{0}=1.76 \times$ $10^{-44} \mathrm{~cm}^{2}, g_{A}=1.257, m_{e}$ is the electron mass and $E_{\nu}$, the neutrino energy. This formula must be actually corrected by a factor of $3-10$ to take into account the degeneracy and Fermi liquid corrections [31], we will use a factor of ten in this paper. Similarly for quark matter we consider the absorption processes of degenerate neutrinos by degenerate down quarks [44]:

$$
\lambda_{q}=\frac{5 \pi^{3} \mu_{\nu}^{2}}{2 G_{F}^{2} \mu_{e}^{3}\left(10 \mu_{u}^{2}+5 \mu_{u} \mu_{e}+\mu_{e}^{2}\right)\left(\left(E_{\nu}-\mu_{\nu}\right)^{2}+\pi^{2} T^{2}\right)}
$$

This formula holds for normal quark matter and it can be used also for the blue quarks of the 2SC phase which are indeed unpaired. We therefore multiply $\lambda_{q}$ by a factor of three within the $2 \mathrm{SC}$ phase to take into account that the absorption process can take place only if blue quarks are involved. For the paired red and green quarks of the 2SC phase, the corresponding mean free path is larger 14] then the one associated with the blue free quarks and thus we can safely neglect them in our calculation. Finally, since we study the regime in which neutrinos are still degenerate, the spectral average of the mean free path is dominated by neutrinos at the Fermi surface [35] thus we set, in Eq. 12-13, $E_{\nu}=\mu_{\nu}$. A last comment about the neutrino mean free path within the mixed phase $\lambda_{M P}$ : it is simply a weighted sum of the mean free paths of the two phases: $1 / \lambda_{M P}=\chi / \lambda_{q}+(1-\chi) / \lambda_{n}$.

Let us discuss now our results by starting from the case of the deleptonization of an ordinary neutron star, which will constitute our reference model. We fix the central pressure of the star $p_{c}=83 \mathrm{MeV} / \mathrm{fm}^{3}$, and calculate the equilibrium configuration for the initial uniform profiles of $S / N$ and $Y_{L}$. We stop the integration of the structure equations when the pressure drops to a value $p_{0}$ corresponding to a baryon density of roughly half of the nuclear saturation density [52]. We obtain a baryon number of $N_{B} \sim 2.29 \times 10^{57}$ with a corresponding gravitational mass of $\sim 1.78 M_{\odot}$. We then start the diffusion simulation [53]. Results for the temporal variations of the neutrino density, the baryon density and the pressure profiles are shown in Figs. 3-5. From Fig. 3 one can notice that while we are here adopting many simplifying assumptions for the transport process, the time scale of deleptonization is in the right order of magnitude: after $t^{*} \sim 7 \mathrm{~s}$ the central neutrino density is half of its initial value. Similar estimates are obtained with the simple treatment of the deleptonization and cooling time scales presented in [31] and in the simulations of [36] (on the other hand, in the more recent one [38], a better treatment of the baryon - neutrino interactions 


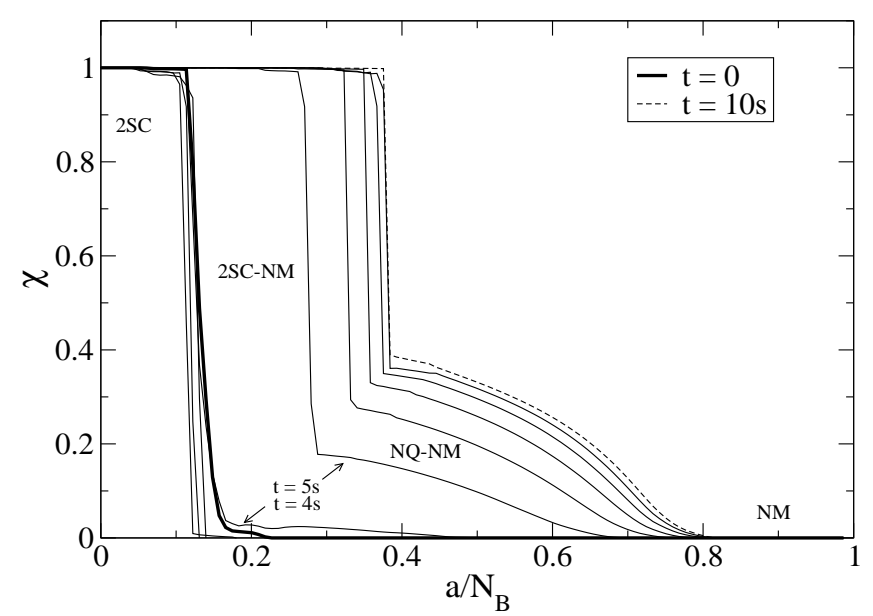

FIG. 7: Temporal evolution of the volume fraction of the quark phase for a hybrid star of baryonic number $N_{B}=2.29 \times$ $10^{57}$. Initially the star has a core of $2 \mathrm{SC}$ phase, a thin layer of mixed phase and an outer layer of nucleonic matter. After $\sim 4 \mathrm{~s}$ a sizable change of the structure of the star occurs due to the formation of the normal quark phase triggered by deleptonization. The mixed phase has three components: nucleonic matter, 2SC and normal quark matter.

leads actually to a deleptonization process which continues through most of the cooling epoch, $\left.t_{\text {del }} \sim 50 \mathrm{~s}\right)$. We stop our simulation at $t=10 \mathrm{~s}$, since for longer times neutrino would start to become non-degenerate and our assumptions do not hold anymore.

Instead, the temporal behavior of the density and the pressure is different from what was found in [36, 38] but in qualitative agreement with the results of [37] (see Figs. 3 and 10 of that paper): during the first seconds of the evolution, while in the outer layers of the star $\left(a / N_{B}\right.$ close to 1) the density increases due to the lack of neutrino pressure, the pressure and density at the center of the star slightly decrease. This is actually a consequence of the stiffening of the nucleonic EoS when $Y_{L}$ is reduced: even if the contribution to the total pressure of neutrinos decreases during deleptonization, nucleonic matter becomes more and more isospin asymmetric and, due to the large value of the symmetry energy at high density within the TM1 model (see [15]), the overall effect is a stiffer EoS. This effect deserves more accurate studies by implementing a different choice for the initial profiles of $Y_{L}$ and $S / N$ taken from supernova simulations and by improving the treatment of the neutrino and energy transport (energy transport is not included here). Interestingly, in the recent sophisticated calculation presented in Ref. [40], the central density is almost constant during the long term evolution (see Fig. 16a of that paper) at variance with the results of [38]. Here we want just to stress that the symmetry energy could play an important role in protoneutron star evolution and, since quark matter has usually a smaller symmetry energy than nucleonic matter, hybrid stars show a different temporal evolution as we will show in the following.

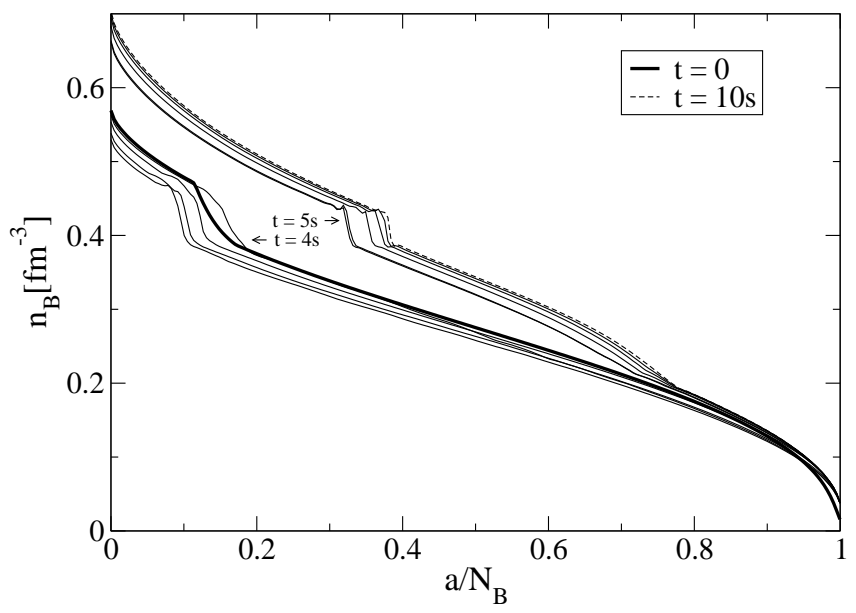

FIG. 8: Temporal variation of the baryon density as a function of the enclosed baryonic number for a hybrid star of baryonic number $N_{B}=2.29 \times 10^{57}$. The thick line corresponds to the initial configuration $t=0$ and the dashed line to the configuration at $t=10 \mathrm{~s}$. The thin solid lines stand for the intermediate configurations with temporal steps of $1 \mathrm{~s}$. The central density slightly decreases during the first seconds but after $\sim 4 \mathrm{~s}$ when the normal quark phase appears, it starts to increase.

By looking at the pressure profiles in Fig. 5, we notice that the value of the pressure at $a=N_{B}$ is the same at all times as it should be, which represents an important check of our calculation.

Let us discuss now the results obtained for the hybrid EoS presented in Sec. II. We consider an hybrid star having the same baryon number as the neutron star considered before. The corresponding central pressure is $p_{c}=118 \mathrm{MeV} / \mathrm{fm}^{3}$ and the initial gravitational mass $M=1.78 M_{\odot}$. After calculating the initial configuration with constant $S / N$ and $Y_{L}$, we start the temporal evolution simulation. The temporal variation of the neutrino density is shown in Fig. 6: notice that the neutrino density profile follows the same shape of the baryon density profile (Fig. 8), with the typical features of a first order phase transition (a mixed phase and two pure phases). In the pure $2 \mathrm{SC}$ quark phase, the density of neutrinos is larger than in the pure nucleonic phase, the central neutrino density is in this case $n_{\nu_{0}}=3.44 \times 10^{-2} \mathrm{fm}^{-3}$. This is due to the depletion of electrons in the quark phase, with respect to the nucleonic phase, and the condition of fixed lepton fraction [43]. The deleptonization time of the hybrid star is comparable with the one of the neutron star: although the mean free path within the quark phase is larger than the one in the nucleonic phase at the same value of baryon density [44], neutrinos enter the nucleonic phase after diffusing inside the quark phase and the mixed phase. Since they are more abundant than in the case of the neutron star, their mean free path within the nucleonic phase is strongly suppressed (see the dependence of $\lambda_{n}$ on $E_{\nu}$ in Eq. (12)). The modification of the structure and the composition of the star is shown in Fig. 7 where the quark volume fraction profile is dis- 
played. This figure represents the main result of this paper. Starting with an initial configuration with a core of 2SC phase, a small region of mixed phase and a nucleonic matter layer, the star gradually modifies its structure and composition during deleptonization, and at some point ( $t \sim 4 \mathrm{~s}$ in this example), close to the onset of the mixed phase, the 2SC pairing pattern is broken and the normal quark phase is formed. The mixed phase has now three components and occupies a much larger volume fraction of the star and continues to grow until a stationary configuration is reached when almost all neutrinos leak out of the star. Quite remarkably, in a short amount of time, between 4 and $5 \mathrm{~s}$ the structure of the star changes sizably with possible observable effects. This change of the structure of the star is also evident from the baryon density profile shown in Fig. 8. The baryon density slightly decreases during the first seconds, similarly to the neutron star case, but after $\sim 4 \mathrm{~s}$, in coincidence with the formation of the normal quark phase in the mixed phase, it starts to increase. Initially only a small fraction of the star is occupied by the 2SC phase and therefore the evolution of the system is dominated by the nucleonic phase. With the formation of the normal quark phase, very quickly a large fraction of the star is occupied by the quark and the mixed phase and the density starts to increase further favoring the formation of the quark phase. This behavior is compatible with the fact that the EoS of the mixed phase and the pure quark phase softens as the lepton fraction decreases (see Fig. 2.). This qualitative difference between neutron stars and stars containing an exotic phase was already found in [37] for the case of hyperonic matter. Although very interesting, because related with the different symmetry energy of the nucleonic phase and the quark phase, the different evolution of the density in neutron stars and hybrid stars must be investigated in more sophisticated transport models and by using more realistic initial conditions before a firm conclusion can be drawn. In the same figure, one can also notice how the profile of the density qualitatively changes during the evolution because of the sizable modification of the mixed phase. Unfortunately, we cannot provide here signatures of this structural and chemical changes in the star within the neutrino signal released; such a calculation represents an important extension of this work.

Let us consider one more case with a smaller protoneutron star total baryon number. Fig. 9 shows the temporal evolution of the neutrino density for a neutron star having an initial gravitational mass of $1.38 M_{\odot}$ and $N_{B}=1.74 \times 10^{57}$. The initial central density is smaller than the density of the onset of the mixed phase, the evolution proceeds as in the case of neutron stars with a slow decrease of the central density and after $t \sim 6 \mathrm{~s}$, when a large part of neutrinos already left the star, the normal quark phase starts to appear in the mixed phase and reaches an equilibrium volume fraction of $\sim 0.2$, shown in the insert of Fig. 9 (this scenario is similar to the cases considered in [43]). In this case, the $2 \mathrm{SC}$ phase

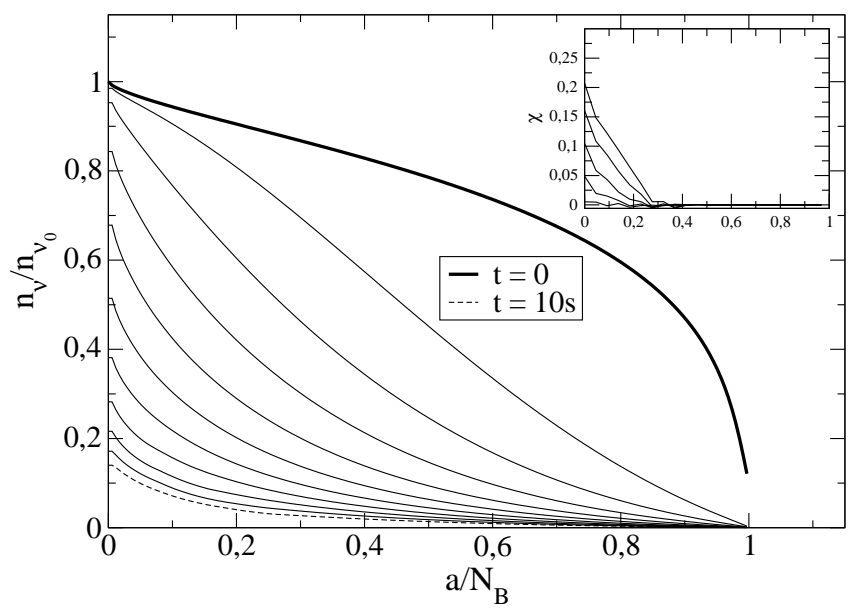

FIG. 9: Temporal variation of the neutrino density as a function of the enclosed baryonic number for a hybrid star of baryonic number $N_{B}=1.74 \times 10^{57}$. The initial central neutrino density, $n_{\nu_{0}}=1.66 \times 10^{-2} \mathrm{fm}^{-3}$. The thick line corresponds to the initial configuration $t=0$ and the dashed line to the configuration at $t=10 \mathrm{~s}$. The thin solid lines stand for the intermediate configurations with temporal steps of $1 \mathrm{~s}$. The insert shows the quark volume fraction profiles for the latest 4 seconds of the evolution. Only the normal quark phase is formed in this case at late stages of the deleptonization process.

cannot be formed because the initial conditions are such that quark matter appears when matter is already very isospin asymmetric.

For very large initial total baryon number, a collapse is obtained during the evolution because the maximum mass is reached. The scenario in that case is quite similar to the one obtained for hyperonic matter, kaon condensed matter and normal quark matter [37 39, 42, 43]. Due to our simple treatment of the initial conditions and the neutrino transport we cannot provide here the evolution of configurations close to the maximum mass since for those cases the initial conditions are crucial for the stability of the star with respect to collapse. A full calculation from the supernova explosion to the late evolution of protoneutron stars is needed for very massive stellar objects as done in [40, 41].

\section{CONCLUSIONS}

We have computed the temporal evolution of protoneutron stars containing a color superconducting phase in the core. We considered here the case of the 2SC phase which, between the different color superconducting phases, is the most likely to appear in a newly born neutron star. During the first few seconds after the birth of the star, the gradual deleptonization is accompanied by a reduction of the electron and proton fractions: matter becomes more and more isospin asymmetric. In the $2 \mathrm{SC}$ quark phase, when the isospin asymmetry reaches a certain threshold the pairing cannot take place anymore and 
a first order phase transition to the normal quark phase occurs. Interestingly, the unpairing transition takes place close to the onset of the nucleonic matter - quark matter mixed phase, where the mismatch between the quark chemical potentials is the highest. The structure and composition of the star are strongly modified during the phase transition and they reach an equilibrium configuration at late times when most of the neutrinos are released. This is the typical evolution of a star with initial gravitational mass larger than $\sim 1.6 M_{\odot}$. For less massive hybrid stars, quark matter appears only at later times and the isospin asymmetry is then so large that the 2SC phase cannot be formed and only a normal quark phase can appear in the core of the star.

The calculations we have presented here are based on many simplifying assumptions for the neutrino interactions and for the treatment of the neutrino transport. We have nevertheless provided a few examples of how the evolution of a protoneutron star can be rich and complex when color superconducting phases are taken into account. More realistic calculations are of course needed and also the three flavor CFL phase should be included in the equation of state. Work along this line is already in progress.

Finally we want to remark why the scenario we have discussed here is potentially very interesting for phenomenology: the neutrino signal, could be actually modified by the happenings of the core of the star as shown in [45 47] for the scenario of a phase transition to quark matter in the early post-bounce stage of a supernova. If sizable modifications of the structure of the star occurs one could indeed expect to have signatures on the spectrum and the luminosity of the emitted neutrinos: for instance, the average energy of the emitted neutrinos strongly depends on the compactness of the star [40]. Also the effect of metastability and nucleation connected with a first order phase transition [48] could have an imprint on the temporal structure of the signal. The present neutrino detectors, as SuperK, will be able to catch thousands of neutrinos for a galactic supernova thus allowing a detailed temporal and spectral investigation of the signal [46].

Another very exciting implication of our scenario concerns gamma-ray-bursts: there are now many hints on the possibility that at least some gamma-ray-bursts are produced by strongly magnetized neutron stars. The magnetar model of gamma-ray-bursts [49] as compared to the collapsar model could explain better the long term activity, up to $10^{6} \mathrm{~s}$ after the prompt emission, seen in some light curves [50, 51]. This implies that the prompt emission is connected with the first stages of the evolution of a protoneutron star and the specific features of the neutrino signal could have therefore an impact on the gamma-ray-bursts. The complex temporal structure of some gamma-ray-bursts could have its explanation in the complex phenomena which can occur in a protoneutron star if quark phases are formed.

This work is supported by the Deutsche Forschungsgemeinschaft (DFG) under Grant No. PA 1780/2-1. The author thanks J. Schaffner-Bielich for valuable discussions. This work was also supported by CompStar, a Research Networking Programme of the European Science Foundation.
[1] M. G. Alford, A. Schmitt, K. Rajagopal, and T. Schafer, Rev. Mod. Phys. 80, 1455 (2008), 0709.4635.

[2] A. W. Steiner, S. Reddy, and M. Prakash, Phys. Rev. D66, 094007 (2002), hep-ph/0205201.

[3] S. B. Ruester, V. Werth, M. Buballa, I. A. Shovkovy, and D. H. Rischke, Phys. Rev. D72, 034004 (2005), hep$\mathrm{ph} / 0503184$.

[4] D. Blaschke, S. Fredriksson, H. Grigorian, A. M. Oztas, and F. Sandin, Phys. Rev. D72, 065020 (2005), hep$\mathrm{ph} / 0503194$.

[5] H. J. Warringa (2006), hep-ph/0606063.

[6] R. Casalbuoni, R. Gatto, N. Ippolito, G. Nardulli, and M. Ruggieri, Phys. Lett. B627, 89 (2005), hep$\mathrm{ph} / 0507247$.

[7] S. B. Ruester, V. Werth, M. Buballa, I. A. Shovkovy, and D. H. Rischke, Phys. Rev. D73, 034025 (2006), hepph/0509073.

[8] F. Sandin and D. Blaschke, Phys. Rev. D75, 125013 (2007), astro-ph/0701772.

[9] G. Lugones, T. A. S. do Carmo, A. G. Grunfeld, and N. N. Scoccola, Phys. Rev. D81, 085012 (2010), 1001.1709 .

[10] A. Drago, A. Lavagno, and G. Pagliara, Phys. Rev. D69, 057505 (2004), nucl-th/0401052.
[11] A. Drago and G. Pagliara, Astrophys. J. 665, 1227 (2007), astro-ph/0512602.

[12] G. Pagliara and J. Schaffner-Bielich, Phys. Rev. D77, 063004 (2008), 0711.1119.

[13] A. Drago, G. Pagliara, G. Pagliaroli, F. L. Villante, and F. Vissani, AIP Conf. Proc. 1056, 256 (2008), 0809.0518.

[14] G. W. Carter and S. Reddy, Phys. Rev. D62, 103002 (2000), hep-ph/0005228.

[15] G. Pagliara and J. Schaffner-Bielich, Phys. Rev. D81, 094024 (2010), 1003.1017.

[16] P. F. Bedaque, Nucl. Phys. A697, 569 (2002), hep$\mathrm{ph} / 9910247$.

[17] O. Kiriyama, S. Yasui, and H. Toki, Int. J. Mod. Phys. E10, 501 (2001), hep-ph/0105170.

[18] S. Lawley, W. Bentz, and A. W. Thomas, Phys. Lett. B632, 495 (2006), nucl-th/0504020.

[19] G. B. Partridge, W. Li, R. I. Kamar, Y. Liao, and R. G. Hulet, Science 311, 503 (2006), arXiv:condmat/0511752.

[20] P. F. Bedaque, H. Caldas, and G. Rupak, Phys. Rev. Lett. 91, 247002 (2003), cond-mat/0306694.

[21] M. Prakash, J. R. Cooke, and J. M. Lattimer, Phys. Rev. D52, 661 (1995).

[22] A. Steiner, M. Prakash, and J. M. Lattimer, Phys. Lett. 
B486, 239 (2000), nucl-th/0003066.

[23] H. Shen, H. Toki, K. Oyamatsu, and K. Sumiyoshi, Nucl. Phys. A637, 435 (1998), nucl-th/9805035.

[24] E. S. Fraga, R. D. Pisarski, and J. Schaffner-Bielich, Phys. Rev. D63, 121702 (2001), hep-ph/0101143.

[25] M. Alford, M. Braby, M. W. Paris, and S. Reddy, Astrophys. J. 629, 969 (2005), nucl-th/0411016.

[26] P. Demorest, T. Pennucci, S. Ransom, M. Roberts, and J. Hessels, Nature 467, 1081 (2010), 1010.5788.

[27] A. Drago and U. Tambini, J. Phys. G25, 971 (1999), astro-ph/9703138.

[28] M. Hempel, G. Pagliara, and J. Schaffner-Bielich, Phys. Rev. D80, 125014 (2009), 0907.2680.

[29] G. Pagliara, M. Hempel, and J. Schaffner-Bielich, J. Phys. G37, 094065 (2010), 1001.2191.

[30] G. Pagliara, M. Hempel, and J. Schaffner-Bielich, Phys. Rev. Lett. 103, 171102 (2009), 0907.3075.

[31] M. Prakash et al., Phys. Rept. 280, 1 (1997), nuclth/9603042.

[32] M. Di Toro, A. Drago, T. Gaitanos, V. Greco, and A. Lavagno, Nucl. Phys. A775, 102 (2006), nuclth/0602052.

[33] M. Di Toro et al. (2009), 0909.3247.

[34] F. Neumann, M. Buballa, and M. Oertel, Nucl. Phys. A714, 481 (2003), hep-ph/0210078.

[35] A. Burrows, T. J. Mazurek, and J. M. Lattimer, Astrophys. J. 251, 325 (1981).

[36] A. Burrows and J. M. Lattimer, Astrophys. J. 307, 178 (1986).

[37] W. Keil and H. T. Janka, Astron. Astrophys. 296, 145 (1995).

[38] J. A. Pons, S. Reddy, M. Prakash, J. M. Lattimer, and J. A. Miralles, Astrophys. J. 513, 780 (1999), astroph/9807040.

[39] T. W. Baumgarte, S. A. Teukolsky, S. L. Shapiro, H. T. Janka, and W. Keil, Astrophys. J. 468, 823 (1996).

[40] T. Fischer, S. C. Whitehouse, A. Mezzacappa, F. K. Thielemann, and M. Liebendorfer, Astron.Astrophys. 517, A80 (2009), 0908.1871.

[41] L. Hudepohl, B. Muller, H. T. Janka, A. Marek, and G. G. Raffelt, Phys. Rev. Lett. 104, 251101 (2010), 0912.0260 .
[42] J. A. Pons, S. Reddy, P. J. Ellis, M. Prakash, and J. M. Lattimer, Phys. Rev. C62, 035803 (2000), nuclth/0003008.

[43] J. A. Pons, A. W. Steiner, M. Prakash, and J. M. Lattimer, Phys. Rev. Lett. 86, 5223 (2001), astro$\mathrm{ph} / 0102015$.

[44] A. W. Steiner, M. Prakash, and J. M. Lattimer, Phys. Lett. B509, 10 (2001), astro-ph/0101566.

[45] I. Sagert et al., Phys. Rev. Lett. 102, 081101 (2009), 0809.4225.

[46] B. Dasgupta et al., Phys. Rev. D81, 103005 (2010), 0912.2568.

[47] T. Fischer et al. (2010), 1011.3409.

[48] B. W. Mintz, E. S. Fraga, G. Pagliara, and J. SchaffnerBielich, Phys. Rev. D81, 123012 (2010), 0910.3927.

[49] B. D. Metzger (2010), 1001.5046.

[50] S. Dall'Osso et al. (2010), 1004.2788.

[51] M. G. Bernardini et al. (2010), 1004.3831.

[52] At lower densities, where the crust takes place, the diffusion approximation does not hold anymore because neutrinos are free streaming and a more sophisticated transport calculation would be needed. Here we want to study only the change of the chemical composition of the core of the star thus we can neglect the low density part of the star.

[53] We use, for solving Eqs. 7-11, an iterative method also used in [38]. We solve at a certain time step Eq. 7 by using an implicit finite difference method (in a time - enclosed baryonic number grid ) and we insert the obtained profile of $Y_{L}$ in the structure equations; we calculate then the equilibrium configuration by using the Runge-Kutta method and we plug back the adjusted values of the thermodynamical and stellar quantities (baryon density, pressure, gravitational mass, radius, etc.) in Eq.7 and we re-calculate its solution for the same temporal step. We repeat this procedure until convergence is reached. An important point in this calculation is the conservation of the total baryon number. This constraint is fulfilled, when solving the structure equations, by changing the value of the central pressure of the star until we obtain $a\left(p_{0}\right)=N_{B}$ at the last Runge-Kutta iteration. 